\title{
CAPÍTULO 47: PADRÃO DE IDENTIDADE E QUALIDADE DO MEL DE APIS MELLIFERA: UMA BREVE REVISÃO
}

\section{CHAPTER 47: IDENTITY AND QUALITY STANDARD OF APIS MELLIFERA HONEY: A BRIEF REVIEW}

\author{
Clara Mariana Gonçalves Lima ${ }^{1}$; Siluana Katia Tischer Seraglio ${ }^{2}$; Greici Bergamo ${ }^{3}$; Roseane Fett ${ }^{4}$; Ana \\ Carolina Oliveira Costa ${ }^{5}$
}

\section{Resumo}

Os parâmetros físico-químicos definidos no Padrão de Identidade e Qualidade (PIQ) dos méis de abelhas Apis mellifera buscam garantir a qualidade e segurança de méis para consumo humano. Portanto, considerando a importância do PIQ dos méis de abelhas Apis mellifera, a proposta dessa breve revisão foi apresentar e discutir os parâmetros físico-químicos necessários para a comercialização segura desse alimento. Sendo assim, foram reportadas informações relacionadas à umidade, açúcares, acidez livre, 5-hidroximetilfurfural, atividade diastásica, cinzas, minerais, condutividade elétrica e sólidos insolúveis. A partir dessas informações, a importância de cada um desses parâmetros na inibição de ações fraudulentas, de processamento mal conduzido, entre outros foram demonstradas. Portanto, essa revisão demostra a importância desses parâmetros mínimos como forma de assegurar a identidade e qualidade do mel, bem como da necessidade de constante fiscalização a fim de evitar riscos à saúde dos consumidores devido ao consumo de um mel não adequado.

Palavras-Chave: Composição, Mel de melato, Mel floral, Parâmetros físico-químicos.

\begin{abstract}
The physicochemical parameters defined in the Identity and Quality Standard (IQS) of Apis mellifera bee honeys aim to guarantee the quality and safety of honeys for human consumption. Therefore, considering the importance of IQS of honeys from Apis mellifera bees, the purpose of this brief review was to present and discuss the physicochemical parameters necessary for the safe marketing of that food. In this sense, information related to moisture, sugars, free acidity, 5-hydroxymethylfurfural, diastatic activity, ashes, minerals, electrical conductivity, and insoluble solids were reported. Based on this information, the importance of each of these parameters in inhibiting fraudulent actions, poorly conducted processing, among others, has been demonstrated. Thus, this review demonstrates the importance of these minimum parameters as a way of ensuring the identity and quality of honey, as well as the need for constant inspection in order to avoid risks to consumers' health due to the consumption of an unsuitable honey.
\end{abstract}

Keywords: Composition, Honeydew honey, Blossom honey, Physicochemical parameters.

\footnotetext{
${ }^{1}$ Doutoranda, Ciências dos Alimentos, Universidade Federal de Santa Catarina, claramarianalima@ gmail.com

${ }^{2}$ Pós-doutoranda (CNPq Scholarship - Brazil (160175/2019-4)), Ciências dos Alimentos, Universidade Federal de Santa Catarina, siluanaseraglio@ hotmail.com

${ }^{3}$ Doutora, Ciências dos Alimentos, Universidade Federal de Santa Catarina, greici.bergamo@ hotmail.com

${ }^{4}$ Professora, Doutora em Química Orgânica, Universidade Federal de Santa Catarina, roseane.fett@ufsc.br

${ }^{5}$ Professora, Doutora em Química Analítica, Universidade Federal de Santa Catarina, ana.costa@ufsc.br
} 


\section{Introdução}

O Brasil possui um notável potencial apícola (CALDAS et al., 2019) devido à diversificada flora melitófila, condições edafoclimáticas favoráveis e ampla extensão territorial (MARQUES et al., 2011).

O mel é um alimento de sabor doce composto por macro e micro nutrientes que conferem propriedades nutricionais e medicinais ao produto (KATEEL et al., 2018; KHALIL et al., 2015), produzido principalmente por abelhas da espécie Apis mellifera. De acordo com sua origem podem ser classificados como floral, quando obtidos a partir do néctar das flores; ou melato, quando obtidos por meio de secreções de plantas ou de excreções de insetos sugadores de plantas (BRASIL, 2000; CODEX ALIMENTARIUS, 2001; EUROPEAN COMMISSION, 2002). Méis de origem floral ainda podem ser classificados como uni/monofloral ou multi/polifloral, de acordo com a origem botânica do néctar utilizado pelas abelhas na produção do mel (BRASIL, 2000; MERCOSUL, 1999).

Como forma de avaliar a qualidade dos méis produzidos e comercializados, alguns parâmetros foram elencados por órgãos regulamentadores e fiscalizadores como forma de avaliar a qualidade do produto (BRASIL, 2000; CODEX ALIMENTARIUS, 2001; EUROPEAN COMMISSION, 2002). Os principais critérios de interesse são a avaliação da maturidade do mel, determinado pelo teor de umidade, açúcares não redutores e redutores; pureza, determinada basicamente pelos teores de sólidos insolúveis, cinzas e condutividade elétrica; e o estado de deterioração determinado pelos parâmetros de acidez livre, diastase e 5hidroximetilfurfural (5-HMF). De acordo com Carvalho et al. (2020), vários estudos demonstraram que uma elevada porcentagem de amostras de mel de diferentes regiões do Brasil não está adequada às normas vigentes que os adequam ao consumo.

Cabe ressaltar que devido à diversidade na composição química dos diferentes tipos de méis, os limites mínimo e máximo de cada parâmetro estipulados pelos principais órgãos regulamentadores podem variar de acordo com determinados fatores, como a origem botânica e o local de produção, por exemplo. Méis de origem floral e de melato, possuem limites diferentes para o teor de cinzas, por exemplo. Méis produzidos em países tropicais cuja temperatura ambiente é maior em relação a países de regiões subtropicais, maiores valores para 5-HMF são admitidos por algumas legislações. Outras exceções também são atribuídas para méis que reconhecidamente possuem atividade enzimática baixa onde menores valores de atividade diastásica são aceitos (BRASIL, 2000; CODEX ALIMENTARIUS, 2001; EUROPEAN COMMISSION, 2002). 
Considerando a importância do Padrão de Identidade e Qualidade do mel para a comercialização desse produto, a proposta dessa breve revisão é apresentar e discutir os parâmetros físico-químicos necessários para garantir a qualidade e segurança alimentar dos méis de Apis mellifera.

\section{Desenvolvimento}

\section{Definição, tipos e descrição breve da composição do mel de Apis mellifera}

O mel é definido como um produto elaborado por abelhas melíferas (Apis mellifera). Este pode ser produzido a partir do néctar de flores, sendo este designado como mel floral, ou principalmente de secreções adocicadas de partes vivas de plantas e/ou excreções de insetos sugadores de plantas, sendo essas secreções/excreções chamadas também de melato, originando assim o mel de melato. Após recolhidas, estas substâncias são transformadas e combinadas com substâncias próprias das abelhas, que armazenam este produto nos favos da colmeia para maturação, originando ao final desse processo o mel propriamente dito (BRASIL, 2000; CODEX ALIMENTARIUS, 2001; EUROPEAN COMMISSION, 2002; MERCOSUL, 1999).

Devido à diversidade de fontes botânicas disponíveis, os méis florais podem ser classificados como unifloral ou monofloral quando provenientes majoritariamente de flores de uma mesma família, gênero ou espécie; ou multifloral ou polifloral quando obtidos a partir de diferentes origens botânicas (BRASIL, 2000; MERCOSUL, 1999). Em relação ao mel de melato, a mistura deste mel com mel floral pode ocorrer naturalmente nas próprias colmeias quando existe disponibilidade de néctar e de melato, combinação que ocorre especialmente nos períodos de início do verão e final do inverno no Brasil (BERGAMO et al., 2018; MARENDA et al., 2011). Esta mistura de méis também é considerada como mel de melato por organizações regulamentadoras como Mercosul e Legislação Brasileira, desde que os Parâmetros de Identidade e Qualidade relacionados ao mel de melato sejam atendidos (BRASIL, 2000; MERCOSUL, 1999).

Ainda, segundo a Legislação Brasileira e do Mercosul, o mel pode ser classificado conforme o procedimento de obtenção do mel do favo em: mel escorrido, obtido pelo escorrimento do mel dos favos desoperculados; mel prensado, obtido por meio da prensagem dos favos; e mel centrifugado, obtido pela centrifugação dos favos desoperculados. Além disso, o mel pode ser classificado segundo a sua apresentação e/ou processamento em mel, sendo este em estado líquido, cristalizado ou semi cristalizado; em mel em favo, quando o mel é comercializado nos favos operculados; em mel com pedaços de favos, quando o mel contém um ou mais pedaços de favos; em mel cristalizado, quando este, devido a processos naturais, 
encontrasse no estado sólido; em mel cremoso, quando este apresenta uma estrutura cristalina e fina podendo ter sido submetido a um processo físico; e em mel filtrado, quando o processo de filtração é empregado mas sem acarretar em prejuízos nutricionais ao produto (BRASIL, 2000; MERCOSUL, 1999).

Porém, independentemente do tipo de mel e suas classificações, este é considerado uma matriz complexa constituído por diversas classes de compostos. Alguns destes são oriundos das abelhas (como aminoácidos e enzimas), outros do néctar, melato ou pólen (como minerais, compostos voláteis e compostos fenólicos), bem como podem ser produzidos durante a maturação e estocagem do mel (como ácidos orgânicos, compostos voláteis e compostos de degradação) (ANKLAM, 1998; DA SILVA et al., 2016; KAROUI et al., 2007).

Os monossacarídeos frutose e glicose são os açúcares predominantes, sendo os compostos majoritários e encontrados em concentrações de até $80 \%$ em peso úmido do mel. $\mathrm{Na}$ sequência, a água é o segundo principal constituinte do mel, encontrado normalmente entre 15 a 20\% em peso úmido. Ainda, diversos compostos minoritários são encontrados como enzimas, carotenoides, ácidos orgânicos, aminoácidos, minerais, componentes aromáticos, vitaminas, lipídios, polifenóis, entre outros, sendo estes constituintes responsáveis por inúmeras características sensoriais, físico-químicas e biológicas atribuídas ao mel (BERGAMO et al., 2018, 2019a, 2019b; CAN et al., 2015; ESCUREDO et al., 2013; FECHNER et al., 2016).

\section{Parâmetros de identidade e qualidade do mel de Apis mellifera}

A fim de garantir a identidade e requisitos mínimos de qualidade e segurança do mel para consumo humano, diferentes organizações regulamentadoras ao redor do mundo tem estabelecido normas que ambos os tipos de méis (floral e melato) devem cumprir para serem considerados aptos para consumo e comercialização.

As características sensoriais de méis, como cor, sabor, aroma e consistência, são previstas por organizações regulamentadoras. Em relação à cor dos méis, estes podem variar de quase incolor a pardo-escuro, enquanto o sabor e aroma devem ser característicos da origem do mel (tipo e origem botânica) e a sua consistência dependerá do estado físico do produto. Notase, assim, que essas características são extremamente diversificadas e dependentes fortemente do tipo do mel e das suas origens botânicas (BRASIL, 2000; CODEX ALIMENTARIUS, 2001; EUROPEAN COMMISSION, 2002; MERCOSUL, 1999), o que impossibilita uma definição mais restrita desses requisitos. Entretanto, essas características detêm grande poder sobre a aceitação de um tipo de mel pelo consumidor (AL et al., 2009; BERGAMO et al., 2019b). Por exemplo, espera-se que méis de melato sejam escuros, pela elevada concentração de minerais, 
e não cristalizados, devido ao menor teor de glicose (SERAGLIO et al., 2019). Já em relação aos méis florais, espera-se que méis de eucalipto e de trigo sarraceno sejam escuros, enquanto que méis de tília e de canola sejam claros, características também relacionadas às concentrações dos minerais presentes nesses produtos (BOBIS et al., 2020; KUŚ et al., 2014).

No entanto, os méis florais e de melato distinguem-se entre si especialmente em relação a algumas características físico-químicas (BERGAMO et al., 2019a, 2019b; CAN et al., 2015; PITA-CALVO; VÁZQUEZ, 2017). Dessa forma, são previstos limites distintos para cada uma dessas classes de méis em relação aos parâmetros de açúcares redutores, sacarose, minerais e condutividade elétrica, como apresentado na Tabela 1 (BRASIL, 2000; CODEX ALIMENTARIUS, 2001; EUROPEAN COMMISSION, 2002; MERCOSUL, 1999). 
Tabela 1. Requisitos físico-químicos de identidade e qualidade para méis florais e de melato.

\begin{tabular}{|c|c|c|c|c|}
\hline \multirow{2}{*}{ Parâmetro } & \multicolumn{2}{|c|}{$\begin{array}{c}\text { Legislação Brasileira e } \\
\text { Mercosul }\end{array}$} & \multicolumn{2}{|c|}{$\begin{array}{c}\text { Codex Alimentarius e Comissão } \\
\text { Europeia }\end{array}$} \\
\hline & Tipo do mel & $\begin{array}{c}\text { Valor } \\
\text { estabelecido }\end{array}$ & Tipo do mel & $\begin{array}{c}\text { Valor } \\
\text { estabelecido }\end{array}$ \\
\hline $\begin{array}{l}\text { Soma de frutose } \mathrm{e} \\
\text { glicose }\left(\mathrm{g} 100 \mathrm{~g}^{-1}\right)\end{array}$ & - & - & $\begin{array}{l}\text { Floral } \\
\text { Melato e misturas } \\
\text { com florais }\end{array}$ & $\begin{array}{l}\text { Mínimo } 60 \\
\text { Mínimo } 45\end{array}$ \\
\hline $\begin{array}{l}\text { Açúcares redutores }(\mathrm{g} \\
\left.100 \mathrm{~g}^{-1}\right)\end{array}$ & $\begin{array}{l}\text { Floral } \\
\text { Melato }\end{array}$ & $\begin{array}{l}\text { Mínimo } 65 \\
\text { Mínimo } 60\end{array}$ & - & - \\
\hline Umidade $\left(\mathrm{g} 100 \mathrm{~g}^{-1}\right)$ & Floral/melato & Máximo 20 & Méis no geral & Máximo 20 \\
\hline Sacarose $\left(\mathrm{g} 100 \mathrm{~g}^{-1}\right)$ & $\begin{array}{l}\text { Floral } \\
\text { Melato }\end{array}$ & $\begin{array}{l}\text { Máximo } 6 \\
\text { Máximo } 15\end{array}$ & Méis no geral & Máximo 5 \\
\hline $\begin{array}{l}\text { Sólidos insolúveis em } \\
\text { água }\left(\mathrm{g} 100 \mathrm{~g}^{-1}\right)\end{array}$ & Floral/melato & Máximo $0,1^{*}$ & $\begin{array}{l}\text { Méis no geral não } \\
\text { prensados }\end{array}$ & Máximo 0,1 \\
\hline $\begin{array}{l}\text { Minerais (Cinzas - } \mathrm{g} \\
\left.100 \mathrm{~g}^{-1}\right)\end{array}$ & $\begin{array}{l}\text { Floral } \\
\text { Melato }\end{array}$ & $\begin{array}{l}\text { Máximo 0,6 } \\
\text { Máximo 1,2 }\end{array}$ & - & - \\
\hline $\begin{array}{l}\text { Condutividade elétrica } \\
\left(\mathrm{mS} \mathrm{cm}^{-1}\right)\end{array}$ & - & - & $\begin{array}{l}\text { Méis no geral } \\
\text { Melato e outros } \\
\text { méis específicos }\end{array}$ & $\begin{array}{l}\text { Máximo } 0,8 \\
\text { Mínimo 0,8 }\end{array}$ \\
\hline $\begin{array}{l}\text { Acidez livre ( } \mathrm{mEq} \mathrm{kg}^{-} \\
\text {1) }\end{array}$ & Floral/melato & Máximo 50 & Méis no geral & Máximo 50 \\
\hline Atividade diastásica & Floral/melato & $\begin{array}{l}\text { Mínimo } 8 * * \\
\text { (unidades } \\
\text { Göthe) }\end{array}$ & $\begin{array}{l}\text { Méis no geral } \\
\text { Méis naturalmente } \\
\text { com baixo teor } \\
\text { enzimático }\end{array}$ & $\begin{array}{c}\text { Mínimo } 8 \\
\text { (unidades } \\
\text { Shade) } \\
\text { Mínimo } 3 * * * \\
\text { (unidades } \\
\text { Shade) }\end{array}$ \\
\hline $\begin{array}{l}\text { 5-Hidroximetil- } \\
\text { furfural }\left(\mathrm{mg} \mathrm{kg}^{-1}\right)\end{array}$ & Floral/melato & Máximo 60 & $\begin{array}{l}\text { Méis no geral } \\
\text { Méis de origem } \\
\text { tropical }\end{array}$ & Máximo 40 \\
\hline $\begin{array}{l}\text { Legenda: * Exceto no mel } \\
\text { direta ao público; ** Méis } \\
\text { sempre que o teor de } 5 \\
\text { hidroximetilfurfural não ex } \\
\text { Fonte: Adaptado de Brasil }\end{array}$ & $\begin{array}{l}\text { mensado, com to } \\
\text { m baixo teor en } \\
\text { droximetilfurfu } \\
\text { ler } 15 \mathrm{mg} \mathrm{kg}^{-1} \text {; } \\
00), \text { Mercosul ( }\end{array}$ & $\begin{array}{l}\text { cia de até } 0,5 \\
\text { tico devem ap } \\
\text { ão exceder } 1 \\
- \text { mili equivale } \\
\text {, Codex Alime }\end{array}$ & $\begin{array}{l}0 \mathrm{~g}^{-1} \text {, unicamente er } \\
\text { tar valor de no míni } \\
\mathrm{gg} \mathrm{kg}^{-1} ; * * * \text { Semp } \\
\text {; } \mathrm{mS}-\text { mili Siemens. } \\
\text { ius }(2001) \text { e Europea }\end{array}$ & $\begin{array}{l}\text { odutos para venc } \\
3 \text { unidades Göth } \\
\text { ue o teor de } \\
\text { mmission }(2002\end{array}$ \\
\hline
\end{tabular}


De acordo com a Tabela 1, nota-se que os parâmetros exigidos são semelhantes entre diferentes organizações regulamentadoras, sendo que a principal diferença é encontrada em relação à determinação de cinzas pela Legislação Brasileira e Mercosul e da condutividade elétrica por outras organizações regulamentadoras internacionais. Além disso, os limites estabelecidos para cada parâmetro também são muito similares, senão iguais, entre as diferentes organizações regulamentadoras. Esses parâmetros trazem importantes informações referentes ao grau de maturidade, de deterioração e de pureza do mel (BRASIL, 2000).

Além das delimitações sensoriais e físico-químicas, outras especificações também são descritas, como: o mel não pode apresentar indícios de fermentação; ter sido adicionado de açúcares, aditivos ou quaisquer substâncias que venham a modificar a sua composição original; conter contaminantes como metais pesados, pesticidas ou outros que sejam proibidos ou estejam acima dos limites previstos em regulamentações especificas; conter substâncias estranhas; ser acondicionado em embalagem não própria para alimentos (BRASIL, 2000; CODEX ALIMENTARIUS, 2001; EUROPEAN COMMISSION, 2002; MERCOSUL, 1999). Caso haja aquecimento brando do mel, este não deve comprometer a sua composição e qualidade, assim como tratamentos químicos e bioquímicos não devem ser utilizado (CODEX ALIMENTARIUS, 2001).

Portanto, especialmente por meio das características físico-químicas, objetiva-se garantir a identidade do mel e a sua qualidade e segurança, inibindo ou minimizando a prática de ações de adulteração da autenticidade do mel (BERGAMO et al., 2019a; CAMINA; PELLERANO; MARCHEVSKY, 2012). As adulterações no mel normalmente ocorrem pela adição de água, amidos e glicoses a fim de promover o aumento do rendimento do produto (EVANGELISTA-RODRIGUES et al., 2005). Em âmbito nacional, a Vigilância Sanitária e o Ministério da Agricultura vêm buscando minimizar as fraudes por meio de coletas de amostras em feiras livres, supermercados e comércios de ruas para a realização de testes que identifiquem a autenticidade do alimento (AGUIAR, 2018). Vale salientar que adulterações causam impacto nos ganhos econômicos dos apicultores (SOBRINO-GREGORIO et al., 2018) bem como de todo o ecossistema (ADENEKAN et al., 2015).

Acrescenta-se que, para a detecção de adulterantes no mel normalmente são empregadas, em conjunto com as análises físico-químicas previstas em legislações, técnicas analíticas mais avançadas como: espectroscopia no infravermelho; espectroscopia Raman; espectroscopia de fluorescência; espectroscopia de ressonância magnética nuclear; cromatografia de troca aniônica de alto desempenho acoplado a detector amperométrico 
pulsado; cromatografia a gás acoplada a espectrometria de massas; entre outras (HE et al., 2020; ZÁBRODSKÁ; VORLOVÁ, 2014).

\section{Maturidade}

\section{Umidade}

A água é o segundo maior constituinte presente nos méis, sendo o seu teor dependente da origem botânica e geográfica, condições edafoclimáticas, época de colheita, grau de maturidade alcançado na colmeia, condições de armazenamento, entre outros (CHIRIFE; ZAMORA; MOTTO, 2006; FECHNER et al., 2016; FLORES; ESCUREDO; SEIJO, 2015). Por esses motivos, a umidade do mel varia consideravelmente, podendo inclusive chegar a teores acima de 20\% (FINOLA; LASAGNO; MARIOLI, 2007; SAXENA; GAUTAM; SHARMA, 2010). De acordo com Marchini, Moreti e Otsuk (2005), uma das principais causas da alta umidade do mel é a colheita precoce de favos imaturos ou condições inadequadas de armazenamento. Por essas razões, esse parâmetro é considerado um indicativo da maturidade de méis.

A água afeta várias características do mel, como viscosidade, cristalização, cor, sabor, solubilidade e conservação (ESCUREDO et al., 2014; FECHNER et al., 2016; LAUX; CAMARA; ROSENKRANTZ, 2011). Dentre essas, a cristalização pode ser destacada, uma vez que tem importante impacto na aceitação de um mel pelo consumidor e sobre a conservação do produto. A cristalização é um fenômeno relacionado com a razão frutose/glicose e também com a razão glicose/umidade. Valores inferiores a 1,7 para a razão entre glicose/umidade indicam uma cristalização lenta ou nula, enquanto valores cima de 2 são considerados indicativos de uma cristalização completa e rápida (DOBRE et al., 2012).

A cristalização do mel, especificadamente da glicose, conduz à redução do teor de sólidos solúveis na fase aquosa, promovendo o aumento da atividade de água daquela fração (TOSI et al., 2008; VENIR; SPAZIANI; MALTINI, 2010). Como consequência dessa condição, a ocorrência de fermentação do mel ao longo da sua estocagem é favorecida (NAYIK; DAR; NANDA, 2015). Isso ocorre basicamente pela ação de microrganismos osmotolerantes sobre os açúcares redutores do mel gerando a formação de ácido acético e água (CHIRIFE; ZAMORA; MOTTO, 2006). Entretanto, valores de atividade de água entorno de 0,5 a 0,7 são encontrados em méis (NAYIK; DAR; NANDA, 2015; SAXENA; GAUTAM; SHARMA, 2010), os quais são considerados satisfatórios para a inibição do crescimento desses microrganismos (CHIRIFE; ZAMORA; MOTTO, 2006). 


\section{Açúcares}

A concentração e, principalmente, o perfil de açúcares de um mel depende fortemente de vários fatores como condições climáticas, origem botânica e geográfica, ação de enzimas, processamento e estocagem, os quais podem auxiliar na discriminação de diferentes méis, incluindo méis de melato e floral (BERGAMO et al., 2019a; DE LA FUENTE et al., 2011; DOBRE et al., 2012).

Os monossacarídeos frutose e glicose (açúcares redutores) são os principais constituintes dos méis, sendo a frutose encontrada geralmente em concentrações levemente superiores a glicose (DOBRE et al., 2012; FINOLA; LASAGNO; MARIOLI, 2007; TORNUK et al., 2013). A soma desses dois açúcares são responsáveis por normalmente 65 a $80 \%$ da constituição do mel (DOBRE et al., 2012; PASCUAL-MATÉ et al., 2018; TORNUK et al., 2013). Porém, menores teores de açúcares redutores (a partir de 45\%) também podem ser encontrados em méis, especialmente em méis de melato (CAN et al., 2015; SAXENA; GAUTAM; SHARMA, 2010). Ainda, outros oligossacarídeos, especialmente di e trissacarídeos, são encontrados em méis, porém em concentrações individuais inferiores aos açúcares majoritários (DE LA FUENTE et al., 2011; PASCUAL-MATÉ et al., 2018).

A composição de açúcares do mel afeta diretamente as suas características de higroscopicidade, viscosidade e cristalização, por exemplo (FECHNER et al., 2016; NAYIK; DAR; NANDA, 2015). A cristalização do mel, apesar de ser um processo natural, é comumente considerado um defeito pelos consumidores (ESCUREDO et al., 2014). Nesse processo, é observada a separação de fases o qual ocorre basicamente devido à baixa solubilidade da glicose (VENIR; SPAZIANI; MALTINI, 2010). Valores abaixo de 1,0 para a razão frutose/glicose são comumente indicativos de uma cristalização rápida, enquanto que valores normalmente acima de 1,3 para a razão frutose/glicose são interpretados como sugestivos de uma cristalização lenta ou inexistente (DOBRE et al., 2012). Considerando que méis de melato comumente apresentam teores de glicose mais baixos quando comparados à méis florais, a ausência ou lenta cristalização desse tipo de mel é uma característica reconhecida dessa classe (SERAGLIO et al., 2019).

Os açúcares redutores e a sacarose (oligossacarídeo não redutor correspondendo normalmente de 2 a $3 \%$ dos carboidratos presentes no mel) também são considerados indicadores da maturidade dos méis, pois a sacarose provinda do néctar ou do melato é convertida especialmente na frutose e glicose por enzimas oriundas das abelhas, como a $\alpha$ - e $\beta$ glicosidase (DE LA FUENTE et al., 2011; MATEO; BOSCH-REIG, 1997). Dessa forma, altos teores de sacarose e baixos teores de açúcares redutores são um indicativo de colheita precoce 
do mel, resultando na não conversão total ou quase total da sacarose, ou de um mel originário de alimentação artificial prolongada das colmeias com xaropes de açúcares (ESCUREDO et al., 2013; SAXENA; GAUTAM; SHARMA, 2010; TORNUK et al., 2013). Essa problemática tem levado vários pesquisadores em todo o mundo a buscarem novas estratégias analíticas para enfrentar o desafio de detectar adulterantes no mel, especialmente aqueles à base de açúcar, os quais não são facilmente detectados (WU et al., 2017).

\section{Deterioração}

Acidez livre

A acidez livre presente no mel é proveniente dos ácidos orgânicos, oriundos da fonte de néctar e/ou secreções e excreções sacarínicas que serão utilizadas na produção de mel pelas abelhas (BERGAMO et al., 2019a; BRUGNEROTTO et al., 2019). Quando o processo de fermentação é iniciado, os açúcares presentes no mel são transformados em ácidos orgânicos por meio de leveduras, e, como consequência, ocorre um aumento na acidez (CAVIA et al., 2007; CHIRIFE; ZAMORA; MOTTO, 2006; FINOLA; LASAGNO; MARIOLI, 2007; TORNUK et al., 2013). Dessa forma, a acidez é um parâmetro relacionado à deterioração (BRASIL, 2000) e ao frescor do mel (KARABAGIAS et al., 2017) e valor máximo de 50 mEq $\mathrm{kg}^{-1}$ são determinados por legislações.

Cabe ressaltar que estudos recentes vem demonstrando que alguns méis de melato possuem naturalmente uma acidez mais elevada, superior a $50 \mathrm{mEq} \mathrm{kg}{ }^{-1}$, mesmo sendo frescos (BERGAMO et al., 2019a; TERRAB; DIEZ; HEREDIA, 2003). Esses estudos demonstraram que a acidez encontrada nesses méis é proveniente da alta quantidade de ácidos orgânicos presentes nessa classe específica de mel, não tendo aparentemente uma relação direta com vias fermentativas de deterioração.

\section{5-Hidroximetilfurfural}

O 5-HMF é um produto intermediário do escurecimento não enzimático formando a partir da redução de açúcares através da reação de Maillard, reações de desidratação de hexoses catalisadas em meio ácido ou por reações de caramelização (FECHNER et al., 2016; MOREIRA et al., 2010; SHAPLA et al., 2018).

Esse composto é formado gradualmente durante o aquecimento ou armazenamento do mel. No entanto, vários fatores afetam a sua ocorrência como o perfil de açúcares, $\mathrm{pH}$, acidez, atividade de água, umidade, além da estocagem prolongada e aquecimento (ANKLAM, 1998; FALLICO; ARENA; ZAPPALA, 2008; FECHNER et al., 2016; TORNUK et al., 2013; YÜCEL; SULTANOGLU, 2013). Portanto, o 5-HMF é considerado um parâmetro indicador 
do frescor do mel e dos procedimentos de manipulação destes (ESCUREDO; SEIJO; FERNÁNDEZ-GONZÁLEZ, 2011; FECHNER et al., 2016). Além disso, esse composto é considerado tóxico em determinadas concentrações (DA SILVA et al., 2016), o que reforça a importância dos limites máximos estipulados para o 5-HMF em méis, o qual varia de 40 a 80 $\mathrm{mg} \mathrm{kg}^{-1}$, dependendo da organização regulamentadora (BRASIL, 2000; CODEX ALIMENTARIUS, 2001; EUROPEAN COMMISSION, 2002; MERCOSUL, 1999). Grigoryan (2015) descreveu o 5-HMF como sendo cancerígeno e citotóxico, o qual o constitui como um dos parâmetros mais críticos da qualidade do mel a ser avaliado.

\section{Atividade diastásica}

A diastase é um conjunto de enzimas ( $\alpha$ - e $\beta$-amilase) secretadas pelas abelhas as quais promovem a hidrolise do amido em monômeros menores. Apesar do néctar e melato não possuírem quantidade relevante de amido, essas enzimas parecem contribuir na digestão do pólen pelas abelhas (DE-MELO et al., 2018; FLANJAK et al., 2016).

A atividade enzimática no mel é variável e depende de vários fatores como a quantidade de saliva contendo enzimas adicionadas ao mel pelas abelhas, a quantidade de néctar ou melato processado, as condições fisiológicas das abelhas e da colmeia, além do aquecimento e estocagem prolongada, fatores estes que decrescem a atividade diastásica dos méis (ÁVILA et al., 2018; DE-MELO et al., 2018; FLANJAK et al., 2016; JUAN-BORRÁS et al., 2014). Assim, esse parâmetro é considerado um dos indicadores do frescor do mel, uma vez que espera-se que méis frescos e não aquecidos tenham a presença dessas enzimas ativas, apesar de alguns méis serem reconhecidos por alguns órgãos regulamentadores como méis com teor naturalmente baixo dessas enzimas, sendo assim aplicado a estes méis o limite mínimo de 3 unidades de diastase desde que o teor de 5 -HMF seja de até $15 \mathrm{mg} \mathrm{kg}^{-1}$. Para os demais méis, estes devem apresentar atividade de no mínimo 8 unidades de diastase (BRASIL, 2000; CODEX ALIMENTARIUS, 2001; EUROPEAN COMMISSION, 2002; MERCOSUL, 1999).

A determinação da atividade diastásica do mel é realizada por meio de análise quantitativa, em que uma unidade diastásica corresponde a atividade enzimática de $1 \mathrm{~g}$ de mel, o qual pode hidrolisar $0,01 \mathrm{~g}$ de amido em $1 \mathrm{~h} \mathrm{a} 40{ }^{\circ} \mathrm{C}$, sendo os resultados expressos em unidades de diastase, Schade ou Göthe (SAKAČ; SAK-BOSNAR, 2012). 


\section{Pureza}

Cinzas, minerais e condutividade elétrica

O teor de cinzas é representado por todo o resíduo inorgânico remanescente da queima de matéria orgânica sendo, portanto, um indicador do conteúdo mineral do mel (SAXENA; GAUTAM; SHARMA, 2010). É considerado um parâmetro de pureza por alguns órgãos regulamentadores (BRASIL, 2000) porque se algumas etapas do beneficiamento do mel forem mal conduzidas, material inorgânico (como sujidades) poderá ser agregado ao produto, aumentando os níveis desse parâmetro (SANTOS; OLIVEIRA, 2013). Ainda, alguns autores relacionam o teor de cinzas com a poluição ambiental (ANKLAM, 1998), evidenciando ainda mais a sua relação com a pureza do produto.

O teor de cinzas pode estar relacionado com a possível origem botânica do mel (SAXENA; GAUTAM; SHARMA, 2010), bem como na diferenciação de méis florais e de melato, uma vez que menores valores são normalmente encontrados em méis florais (FINOLA; LASAGNO; MARIOLI, 2007). Baseado nessas evidências, a Legislação Brasileira preconiza teores máximos diferenciados de cinzas para méis florais e de melato $\left(0,6\right.$ e 1,2 g $100 \mathrm{~g}^{-1}$; respectivamente). Valores acima do preconizado podem indicar excesso de sujidades ou poluição ambiental.

Internacionalmente, para fins de avaliação do padrão de identidade e qualidade do mel, o teor de cinzas foi substituído pela condutividade elétrica, uma vez que esse parâmetro é influenciado pela quantidade de minerais, acidez, presença de íons, ácidos orgânicos e proteínas no mel (ALQARNI et al., 2014; CAN et al., 2015; FECHNER et al., 2016; SAXENA; GAUTAM; SHARMA, 2010; YÜCEL; SULTANOGLU, 2013). Assim, da mesma forma que o conteúdo de cinzas, legislações também estipulam limites diferentes para méis florais e de melato (máximo de $0,8 \mathrm{mS} \mathrm{cm} \mathrm{cm}^{-1}$ e mínimo de $0,8 \mathrm{mS} \mathrm{cm}^{-1}$; respectivamente) (CODEX ALIMENTARIUS, 2001; EUROPEAN COMMISSION, 2002).

\section{Sólidos insolúveis}

Os sólidos insolúveis são avaliados como parâmetro de identidade e qualidade do mel pois estão relacionados a resíduos como cera, patas e asas das abelhas e outras sujidades provenientes do campo (MENDES et al., 2009) ou do processamento inadequado (SANTOS; OLIVEIRA, 2013; SILVA et al., 2009). Por estarem relacionados diretamente com resíduos, os principais órgãos regulamentadores determinam limites de máximos para esse parâmetro $(0,1$ g $100 \mathrm{~g}^{-1}$ para méis florais e de melato) (BRASIL, 2000; CODEX ALIMENTARIUS, 2001; EUROPEAN COMMISSION, 2002; MERCOSUL, 1999). Segundo Schlabitz, Silva e Souza 
(2010), os apicultores poderiam remover tais resíduos utilizando uma peneira de malha com orifícios menores que a utilizada comumente.

\section{Considerações finais}

Nessa revisão bibliográfica foram apresentados e discutidos os parâmetros físicoquímicos mínimos necessários para garantir a qualidade e segurança alimentar de méis florais e de melato de abelhas Apis mellifera. Com base nas informações levantas foi possível entender a importância de cada parâmetro exigido por organizações regulamentadoras na limitação da ocorrência de fraudes, de processos mau conduzidos, entre outros. Assim, foi possível identificar que, dentre os parâmetros previstos em legislações, o perfil e concentração de açúcares são os principais indicadores de adulterações de méis por xaropes, por exemplo. Entretanto, todos os demais parâmetros exigidos contribuem com informações relevantes quanto a qualidade e segurança desse produto. Sabe-se que essas análises apresentam limitações quanto ao grau de detecção de alterações, entretanto estas representam uma importante barreira no controle da qualidade de méis. Portanto, considerando que a prática de adulterações em méis é recorrente, torna-se extremamente importante conhecer os parâmetros mínimos envolvidos na garantia da identidade e qualidade do mel, bem como que a fiscalização por parte das autoridades competentes seja realizada de forma constante a fim de banir tais ocorrências.

\section{Agradecimentos}

Os autores gostariam de agradecer o Conselho Nacional de Desenvolvimento Científico e Tecnológico ( $\mathrm{CNPq})$ e a Coordenação de Aperfeiçoamento de Pessoal de Nível Superior (CAPES; código de financiamento 001) pelas bolsas de estudo e suporte financeiro.

\section{Referências}

ADENEKAN, M. O. et al. Physicochemical and vitamin constituents of honey samples obtained from different agro-ecological Zones of Nigeria. Journal of Multidisciplinary Engineering Science Studies, v. 1, n. 1, p. 36-41, 2015.

AGUIAR, A. C. S. Panorama e perspectivas da cadeia produtiva do mel no Brasil. [s.l.] Universidade Federal de Uberlândia, Patos de Minas, 2018.

AL, M. L. et al. Physico-chemical and bioactive properties of different floral origin honeys from Romania. Food Chemistry, v. 112, n. 4, p. 863-867, 15 fev. 2009.

ALQARNI, A. S. et al. Mineral content and physical properties of local and imported honeys in Saudi Arabia. Journal of Saudi Chemical Society, v. 18, n. 5, p. 618-625, 2014.

ANKLAM, E. A review of the analytical methods to determine the geographical and botanical 
origin of honey. Food Chemistry, v. 63, n. 4, p. 549-562, dez. 1998.

ÁVILA, S. et al. Stingless bee honey: Quality parameters, bioactive compounds, healthpromotion properties and modification detection strategies. Trends in Food Science \& Technology, v. 81, n. March, p. 37-50, nov. 2018.

BERGAMO, G. et al. Mineral profile as a potential parameter for verifying the authenticity of bracatinga honeydew honeys. LWT, v. 97, p. 390-395, nov. 2018.

BERGAMO, G. et al. Physicochemical characteristics of bracatinga honeydew honey and blossom honey produced in the state of Santa Catarina: An approach to honey differentiation. Food Research International, v. 116, p. 745-754, fev. 2019a.

BERGAMO, G. et al. Differentiation of honeydew honeys and blossom honeys: a new model based on colour parameters. Journal of Food Science and Technology, v. 56, n. 5, p. 27712777, 10 maio 2019b.

BOBIS, O. et al. Eucalyptus honey: Quality parameters, chemical composition and healthpromoting properties. Food Chemistry, v. 325, n. April, p. 126870, 2020.

BRASIL. Mapa. Ministério da Agricultura, Pecuária e Abastecimento. Instrução Normativa $n^{\circ}$ 11, de 20 de outubro de 2000. Aprova Regulamento técnico de Identidade e Qualidade do Mel. p. $1-4,2000$.

BRUGNEROTTO, P. et al. A capillary electrophoresis method to determine aliphatic organic acids in bracatinga honeydew honey and floral honey. Journal of Food Composition and Analysis, v. 82, p. 103243, set. 2019.

CALDAS, F. et al. COMPOSIÇÃO QUÍMICA, ATIVIDADE ANTIRADICALAR E ANTIMICROBIANA DO PÓLEN APÍCOLA DE FABACEAE. Química Nova, v. 42, n. 1, p. 49-56, 2019.

CAMINA, J. M.; PELLERANO, R. G.; MARCHEVSKY, E. J. Geographical and botanical classification of honeys and apicultural products by chemometric methods. A review. Current Analytical Chemistry, v. 8, n. 3, p. 408-425, 1 maio 2012.

CAN, Z. et al. An investigation of Turkish honeys: their physico-chemical properties, antioxidant capacities and phenolic profiles. Food Chemistry, v. 180, p. 133-141, ago. 2015.

CARVALHO, R. D. A. et al. Assessment of adulteration and mycoflora identification of honey samples marketed in the metropolitan region of Belo Horizonte, Brazil. Research, Society and Development, v. 9, n. 7, p. 440974246, 21 maio 2020.

CAVIA, M. M. et al. Evolution of acidity of honeys from continental climates: influence of induced granulation. Food Chemistry, v. 100, n. 4, p. 1728-1733, jan. 2007.

CHIRIFE, J.; ZAMORA, M. C.; MOTTO, A. The correlation between water activity and \% moisture in honey: fundamental aspects and application to Argentine honeys. Journal of Food Engineering, v. 72, n. 3, p. 287-292, fev. 2006.

CODEX ALIMENTARIUS. Revised Codex Standard for Honey, Standards and Standard Methods. Codex Alimentarius Commission FAO/OMS, v. 11, n. 1987, p. 1-7, 2001. 
DA SILVA, P. M. et al. Honey: Chemical composition, stability and authenticity. Food Chemistry, v. 196, n. April, p. 309-323, abr. 2016.

DE-MELO, A. A. M. et al. Composition and properties of Apis mellifera honey: A review. Journal of Apicultural Research, v. 57, n. 1, p. 5-37, 23 jan. 2018.

DE LA FUENTE, E. et al. Carbohydrate composition of Spanish unifloral honeys. Food Chemistry, v. 129, n. 4, p. 1483-1489, dez. 2011.

DOBRE, I. et al. Rheological behavior of different honey types from Romania. Food Research International, v. 49, n. 1, p. 126-132, nov. 2012.

ESCUREDO, O. et al. Nutritional value and antioxidant activity of honeys produced in a European Atlantic area. Food Chemistry, v. 138, n. 2-3, p. 851-856, jun. 2013.

ESCUREDO, O. et al. Contribution of botanical origin and sugar composition of honeys on the crystallization phenomenon. Food Chemistry, v. 149, p. 84-90, abr. 2014.

ESCUREDO, O.; SEIJO, M. C.; FERNÁNDEZ-GONZÁLEZ, M. Descriptive analysis of Rubus honey from the north-west of Spain. International Journal of Food Science \& Technology, v. 46, n. 11, p. 2329-2336, nov. 2011.

EUROPEAN COMMISSION. European Commission Council Directive 2001/110/EC of 20 December 2001 relating to honey. Official Journal of the European Communities, p. 10-47, 2002.

EVANGELISTA-RODRIGUES, A. et al. Análise físico-química dos méis das abelhas Apis mellifera e Melipona scutellaris produzidos em regiões distintas no Estado da Paraíba. Ciência Rural, v. 35, n. 5, p. 1166-1171, out. 2005.

FALLICO, B.; ARENA, E.; ZAPPALA, M. Degradation of 5-hydroxymethylfurfural in honey. Journal of Food Science, v. 73, n. 9, p. C625-C631, nov. 2008.

FECHNER, D. C. et al. Multivariate classification of honeys from Corrientes (Argentina) according to geographical origin based on physicochemical properties. Food Bioscience, v. 15, p. 49-54, set. 2016.

FINOLA, M. S.; LASAGNO, M. C.; MARIOLI, J. M. Microbiological and chemical characterization of honeys from central Argentina. Food Chemistry, v. 100, n. 4, p. 16491653, jan. 2007.

FLANJAK, I. et al. Croatian produced unifloral honeys characterised according to the protein and proline content and enzyme activities. Journal of Apicultural Science, v. 60, n. 1, p. 3948, 2016.

FLORES, M. S. R.; ESCUREDO, O.; SEIJO, M. C. Assessment of physicochemical and antioxidant characteristics of Quercus pyrenaica honeydew honeys. Food Chemistry, v. 166, p. 101-106, 2015.

GRIGORYAN, K. Safety of honey. In: Regulating Safety of Traditional and Ethnic Foods. 1st ed. ed. Cambridge, USA: Academic Press, 2015. p. 217-246. 
HE, Y. et al. Detection of adulteration in food based on nondestructive analysis techniques: a review. Critical Reviews in Food Science and Nutrition, v. 0, n. 0, p. 1-21, 16 jun. 2020.

JUAN-BORRÁS, M. et al. Effect of country origin on physicochemical, sugar and volatile composition of acacia, sunflower and tilia honeys. Food Research International, v. 60, p. 8694, jun. 2014.

KARABAGIAS, I. K. et al. Characterization and geographical discrimination of commercial $<$ em $>$ Citrus $</$ em $>$ spp. honeys produced in different Mediterranean countries based on minerals, volatile compounds and physicochemical parameters, using chemometrics. Food Chemistry, v. 217, p. 445-455, fev. 2017.

KAROUI, R. et al. The use of front face fluorescence spectroscopy to classify the botanical origin of honey samples produced in Switzerland. Food Chemistry, v. 101, n. 1, p. 314-323, jan. 2007.

KATEEL, R. et al. Antibacterial action of Tropical honey on various bacteria obtained from diabetic foot ulcer. Complementary Therapies in Clinical Practice, v. 30, p. 29-32, fev. 2018.

KHALIL, M. I. et al. Cardioprotective effects of Tualang Honey: amelioration of cholesterol and cardiac enzymes levels. BioMed Research International, v. 2015, p. 286051, maio 2015.

KUŚ, P. M. et al. Antioxidant activity, color characteristics, total phenol content and general HPLC fingerprints of six Polish unifloral honey types. LWT - Food Science and Technology, v. 55, n. 1, p. 124-130, 2014.

LAUX, D.; CAMARA, V. C.; ROSENKRANTZ, E. $\alpha$-Relaxation in honey study versus moisture content: high frequency ultrasonic investigation around room temperature. Journal of Food Engineering, v. 103, n. 2, p. 165-169, mar. 2011.

MARCHINI, L. C.; MORETI, A. C. DE C. C.; OTSUK, I. P. Análise de agrupamento, com base na composição físico-química, de amostras de méis produzidos por Apis mellifera L. no Estado de São Paulo. Ciência e Tecnologia de Alimentos, v. 25, n. 1, p. 8-17, mar. 2005.

MARENDA, T. A. et al. Desenvolvimento de um sistema para classificação do mel em floral e melato por parâmetros físico químicos combinados com redes neurais artificiais. Evidência, v. 11, n. 1, p. 61-74, 2011.

MARQUES, L. J. P. et al. Levantamento da flora apícola em Santa Luzia do Paruá, Sudoeste da Amazônia, Maranhão. Acta Botanica Brasilica, v. 25, n. 1, p. 141-149, mar. 2011.

MATEO, R.; BOSCH-REIG, F. Sugar profiles of Spanish unifloral honeys. Food Chemistry, v. 60, n. 1, p. 33-41, set. 1997.

MENDES, C. D. G. et al. As análises de mel: revisão. Revista Caatinga, v. 22, n. 2, p. 7-14, 2009.

MERCOSUL. Regulamento Técnico MERCOSUL "Identidade e Qualidade do Mel". MERCOSUL/GMC/RES. No 89/99. p. 1-6, 1999.

MOREIRA, R. F. A. et al. Chemical changes in the volatile fractions of Brazilian honeys during 
storage under tropical conditions. Food Chemistry, v. 121, n. 3, p. 697-704, ago. 2010.

NAYIK, G. A.; DAR, B. N.; NANDA, V. Physico-chemical, rheological and sugar profile of different unifloral honeys from Kashmir valley of India. Arabian Journal of Chemistry, ago. 2015 .

PASCUAL-MATÉ, A. et al. Sugar composition and sugar-related parameters of honeys from the northern Iberian Plateau. Journal of Food Composition and Analysis, v. 74, p. 34-43, 2018.

PITA-CALVO, C.; VÁZQUEZ, M. Differences between honeydew and blossom honeys: a review. Trends in Food Science \& Technology, v. 59, p. 79-87, jan. 2017.

SAKAČ, N.; SAK-BOSNAR, M. A rapid method for the determination of honey diastase activity. Talanta, v. 93, p. 135-138, maio 2012.

SANTOS, D. C.; OLIVEIRA, E. N. A. Características físico-químicas e microbiológicas de méis de Apis mellifera L. provenientes de diferentes entrepostos. Comunicata Scientiae, v. 4, n. 1, p. 67-74, 2013.

SAXENA, S.; GAUTAM, S.; SHARMA, A. Physical, biochemical and antioxidant properties of some Indian honeys. Food Chemistry, v. 118, n. 2, p. 391-397, jan. 2010.

SCHLABITZ, C.; DA SILVA, S. A. F.; DE SOUZA, C. F. V. Avaliação de paraâmteros físicoquímicos microbiológicos em mel. Revista Brasileira de Tecnologia Agroindustrial, v. 4, n. 1, p. 80-90, 9 jul. 2010.

SERAGLIO, S. K. T. et al. An overview of physicochemical characteristics and healthpromoting properties of honeydew honey. Food Research International, v. 119, p. 44-66, maio 2019.

SHAPLA, U. M. et al. 5-Hydroxymethylfurfural (HMF) levels in honey and other food products: effects on bees and human health. Chemistry Central Journal, v. 12, n. 1, p. 1-18, 2018.

SILVA, K. D. F. N. L. et al. Características físico-químicas de mel produzido em Limoeiro do Norte durante o armazenamento. Revista Caatinga, v. 22, n. 4, p. 246-254, 2009.

SOBRINO-GREGORIO, L. et al. Monitoring honey adulteration with sugar syrups using an automatic pulse voltammetric electronic tongue. Food Control, v. 91, p. 254-260, set. 2018.

TERRAB, A.; DIEZ, M. J.; HEREDIA, F. J. Palynological, physico-chemical and colour characterization of Moroccan honeys: I. River red gum (Eucalyptus camaldulensis Dehnh) honey. International Journal of Food Science and Technology, v. 38, n. 4, p. 379-386, abr. 2003.

TORNUK, F. et al. Quality characterization of artisanal and retail Turkish blossom honeys: determination of physicochemical, microbiological, bioactive properties and aroma profile. Industrial Crops and Products, v. 46, p. 124-131, abr. 2013.

TOSI, E. et al. Honey diastase activity modified by heating. Food Chemistry, v. 106, n. 3, p. 883-887, fev. 2008. 
VENIR, E.; SPAZIANI, M.; MALTINI, E. Crystallization in "Tarassaco" Italian honey studied by DSC. Food Chemistry, v. 122, n. 2, p. 410-415, set. 2010.

WU, L. et al. Recent advancements in detecting sugar-based adulterants in honey - A challenge. TrAC Trends in Analytical Chemistry, v. 86, p. 25-38, jan. 2017.

YÜCEL, Y.; SULTANOGLU, P. Characterization of honeys from Hatay Region by their physicochemical properties combined with chemometrics. Food Bioscience, v. 1, p. 16-25, mar. 2013.

ZÁBRODSKÁ, B.; VORLOVÁ, L. Adulteration of honey and available methods for detection - a review. Acta Veterinaria Brno, v. 83, n. 10, p. S85-S102, 2014. 